\title{
CHANGES IN REPORTED PHYSICAL HEALTH SYMPTOMS AND SOCIAL FUNCTION WITH PROLONGED EXPOSURE THERAPY FOR CHRONIC POSTTRAUMATIC STRESS DISORDER
}

\author{
Sheila A.M. Rauch, Ph.D., ${ }^{1,2 *}$ Tania E.E. Grunfeld, Grad.Dip.Ed.Psych, ${ }^{3,4}$ Elna Yadin, Ph.D., ${ }^{3}$ \\ Shawn P. Cahill, Ph.D., ${ }^{3}$ Elizabeth Hembree, Ph.D., ${ }^{3}$ and Edna B. Foa, Ph.D. ${ }^{3}$
}

\begin{abstract}
Background: Postraumatic stress disorder (PTSD) is associated with significant bealth risk, illness, and functional impairment, e.g., Green and Kimerling [2004: Physical Health Consequences of Exposure to Extreme Stress. Washington, DC: American Phychological Association] Kimerling et al. [2000: Trauma and Health: $\mathcal{F}$ Trauma Stress 13:115-128]. Methods: These analyses examined whether negative bealth perceptions and general social functioning change with treatment of chronic PTSD among women from a randomized controlled study comparing prolonged exposure (PE; $n=48)$ or PE combined with cognitive restructuring (PE/CR; $n=40)$ to waitlist ( $n=19$; Foa et al., 2005: 7 Consult Clin Psychol 73:953-964]. Results: Self- reported physical bealth difficulties were significantly reduced in the PE and PE/CR conditions compared to the waitlist condition. These reductions did not demonstrate significant change during the 12 month follow-up period. Self-reported discomfort associated with physical bealth difficulties did not demonstrate significant change over treatment. No difference was detected between the active treatment and waitlist conditions. Both the PE and PE/CR groups reported improved social functioning at post treatment compared to the waitlist. Additional improvement in general social functioning was found between 3 and 12 month follow-up assessments. Changes in PTSD and depressive symptoms over treatment accounted for $29 \%$ of the variance in reduction of reported bealth problems and $30 \%$ of the variance in improvement of general social functioning. Importantly, only changes in PTSD symptoms significantly contribute to the model predicting change in physical bealth problems with depression associated only at a trend level. However, collinearity between PTSD and depression makes interpretation difficult. Conclusions: Negative bealth perceptions and general social function improve with PE. Changes in depression and PTSD with treatment are related to these changes. Depression and Anxiety 26:732-738, 2009. (c) 2008 Wiley-Liss, Inc.
\end{abstract}

Key words: treatment; trauma; women; sexual assault; anxiety

${ }^{1}$ Veterans Administration Ann Arbor Healthcare System, Ann Arbor, Michigan

${ }^{2}$ Department of Psychiatry, University of Michigan Medical School, Ann Arbor, Michigan

${ }^{3}$ Department of Psychiatry, School of Medicine, University of Pennsylvania, Philadelphia, Pennsylvania [all work completed at Department of Psychiatry, School of Medicine, University of Pennsylvania, Philadelphia, Pennsylvania.]

${ }^{4}$ RMIT University, Melbourne, Australia
Contract grant sponsor: National Institute of Mental Health; Contract grant number: MH42178.

*Correspondence to: Sheila A.M. Rauch, Ph.D., VA Ann Arbor Healthcare System, 2215 Fuller Road (116c), Ann Arbor, MI 48105. E-mail: sherauch@med.umich.edu

Received for publication 5 October 2007; Revised 20 June 2008; Accepted 24 June 2008

DOI 10.1002/da.20518

Published online 9 September 2008 in Wiley InterScience (www.interscience.wiley.com).

(C) 2008 Wiley-Liss, Inc. 


\section{Introduction}

$\mathbf{T}_{\mathrm{r}}$ aumatic experiences are common occurrences in the general population, not confined to high-risk groups such as combat soldiers or rescue personnel. Estimates of the prevalence of trauma in the population vary widely (between 39 and $90 \%$ ) based on assessment methods and populations assessed. ${ }^{[1-5]}$. The impact of traumatic experiences can include posttraumatic stress disorder (PTSD) and other psychiatric conditions (depression, panic disorder, etc.). However, posttraumatic reactions create a heavy burden on the entire health-care system, as sufferers often present to nonpsychiatric medical settings with a variety of physical complaints before, in lieu of, or in addition to seeking specific psychiatric help. Thus, the current analyses examine beyond symptom change to more global measures of change with treatment. Specially, we examine two important global measures of change: general health perceptions and global social function.

PTSD is associated with significant health risk and illness. ${ }^{[6-8]}$ Indeed, when examining the National Comorbidity sample, Kimerling found that women and men with PTSD were more than twice as likely to have a nonpsychiatric medical condition than those without PTSD even after controlling for age, socioeconomic status, and depression. ${ }^{[9]}$ Zoellner and colleagues found that more severe PTSD symptoms were related to poorer physical health in female victims of sexual assault. ${ }^{[10]}$ Further, PTSD severity predicted reported physical health symptoms beyond negative life events, anger, and depression. Similarly, Rauch and colleagues found that both PTSD and depression severity uniquely and significantly predicted more negative health perceptions among a sample of older adults in primary care. ${ }^{[11]}$ Among a sample of Canadian peacekeeping veterans, Richardson and colleagues found that those veterans with "probable" PTSD reported significantly more primary and specialty medical service use visits $(M=16.4$ visits) in the past year then those without PTSD $(M=6.0$ visits $) .{ }^{[12]}$

In a review of research on the relationship between PTSD and physical health, Schnurr and Green had proposed that PTSD mediates the relationship between exposure to a traumatic experience and the deleterious effects on physical health. ${ }^{[13]}$ In a sample of female sexual assault survivors, PTSD and depression each significantly and uniquely contributed to regression models predicting global health perceptions and severity of physical health problems even after controlling for history of sexual victimization, assault severity, and physical reactions during the assault. ${ }^{[14]}$ Wagner and colleagues found that PTSD severity assessed immediately upon returning from the first Gulf War significantly predicted health problems at 18-24 months even after controlling for combat exposure. ${ }^{[15]}$ Although these data support a unique contribution of PTSD to physical health, determination of true mediation was not possible. In a study that used accepted statistical standards for mediation ${ }^{[16]}$ to examine whether PTSD symptoms mediate the relationship between trauma exposure and physical health difficulties among female Vietnam veterans, PTSD symptom severity mediated this relationship. ${ }^{[7]}$ PTSD severity also mediated the relationship between combat exposure and physical health status in a sample of older veterans. ${ }^{[17]}$

As previously mentioned, depression also appears to have a unique contribution to more negative physical health perceptions among trauma survivors. Although research in this area often focuses on PTSD, some studies have suggested that depression also plays a key role in negative health perceptions. In a sample of older adults in primary care ${ }^{[11]}$ and in a study of female sexual assault survivors, ${ }^{[14]}$ depression significantly contributed to negative health perceptions. However, additional study of this relationship is needed to clarify the roles of PTSD and depression in negative physical health perceptions.

Global social function represents another measure of generalized change with treatment. Many studies have demonstrated reductions in PTSD and related psychopathology with treatment. However, few studies have examined whether these changes are related to changes in general social functioning. As this is a more global measure of functioning, demonstrating functional improvement with treatment and examining whether these improvements are maintained over time allows us to examine the scope of change with $\mathrm{PE}$.

Although much research has begun to examine the relationship between PTSD and physical health, no study to date has examined whether treatment of PTSD results in changes in physical health. Thus, this study examined whether negative health perceptions are reduced and general social functioning is improved with treatment of PTSD among a sample of women with chronic PTSD. We hypothesized that the frequency of reported physical health difficulties as well as the discomfort related to these difficulties will be reduced whereas general social functioning will be improved at posttreatment for those women who received either $\mathrm{PE}$ or $\mathrm{PE} / \mathrm{CR}$ compared to waitlist. We hypothesized that these changes will be maintained through the follow-up period. Second, the relationships between changes in physical health perceptions, global social functioning and changes in PTSD, and depressive symptoms were examined. Given previous research, we hypothesized that reduction in PTSD and depressive symptoms will be related to less physical health difficulties as well as less discomfort related to those physical problems reported. In addition, we hypothesized that reductions in depressive and PTSD symptoms will be related to improved general social functioning. 


\section{MATERIALS AND METHODS}

\section{Participants and Procedures}

Participants were female physical or sexual assault survivors from a published randomized controlled study comparing prolonged exposure alone (PE; $n=48)$ or $\mathrm{PE}$ combined with cognitive restructuring (PE/CR; $n=40$ ) to waitlist (WL; $n=19 ; ;^{[18]}$ ). Please refer to the main article for a complete description of procedures and recruitment. ${ }^{[18]}$ Rates of drop out differed between the WL (3.8\%) and active treatments (PE, 34.2\%; PE/CR, 40.5\%) but not between the treatments (see ${ }^{[18]}$ for comparisons of drop out and completers). These rates of drop out are similar to those found in other PTSD treatment studies. ${ }^{[19]}$ Results from the study ${ }^{[18]}$ indicated that treatment was superior to waitlist, but the two treatments did not differ from one another in reduction of PTSD, depressive, or general anxiety symptom severity. Table 1 presents the demographics for the sample. No significant differences in demographics between the groups were detected. The study was performed in compliance with the Code of Ethics of the World Medical Association and was approved by the University of Pennsylvania Internal Review Board.

The current analyses examined two secondary outcome measures: Pennebaker Inventory of Limbic Languidness (PILL) and the Social Adjustment Scale. ${ }^{[20]}$ As secondary outcome measures, drop out assessments did not include these measures; therefore, only those pts classified as completers will be examined. For the analysis of followup (FU), last observation carried forward (LOCF) was used. In the recent Institute of Medicine report, LOCF was criticized as a method of managing missing data with the assertion that study quality is reduced as the percentage of dropout increases with severe implications if the percentage of missing data exceeds $30 \% .^{[21]}$ However, across the 1 year follow-up period, we observed a $25 \%$ rate of drop with all drops occurring earlier to the 6 month follow-up assessment.
Assessments. Independent evaluations were conducted at pretreatment and posttreatment for all participants and 3-, 6-, and 12months posttreatment for all active treatment. Follow-up assessments were not completed on waitlist participants as most completed treatment after the waitlist period was complete. All evaluations were conducted by trained doctoral or master's level clinicians who were blind to study condition. Participants were instructed by their therapists and the evaluators to not reveal any information that might unblind the evaluator to treatment condition.

Treatments. Female therapists delivered all treatments in individual sessions lasting $90-120 \mathrm{~min}$. In the startup phase of the study, all therapists were trained together in a 5-day workshop led by Edna B. Foa and Constance V. Dancu (PE) and a second 5-day workshop led by David M. Clark (CR). Therapists were trained to use manuals that described the procedures for each session in great detail. All therapists received ongoing supervision throughout the study.

Definition of Dropout and Establishing Groups. Foa et al. ${ }^{[18]}$ utilized a flexible dosing rule in which participants whose selfreported PTSD severity decreased by $70 \%$ or greater by session 8 terminated treatment at session 9. The remaining participants were offered additional treatment up to a total of 12 sessions. Because of this rule, the number of treatment sessions after session 9 was linked to treatment response. Therefore, following Foa et al ${ }^{[18]}$ treatment completers were defined as those participants who attended at least eight sessions (the point at which the decision was made to terminate or continue treatment). In addition, as the PILL frequency subscale is the primary measure of interest for these analyses, only those women who had the PILL frequency subscale for both pretreatment and posttreatment assessments were included in these analyses.

\section{Measures}

Modified Pennebaker Inventory of Limbic Languidness $\left(\mathbf{P I L L}^{[22]}\right)$. This 77 -item version of the measure assesses physical

TABLE 1. Sample Demographics

\begin{tabular}{|c|c|c|c|c|c|}
\hline & WL & $\mathrm{PE}$ & $\mathrm{PE} / \mathrm{CR}$ & $\chi^{2}(\mathrm{df})$ & Overall \\
\hline \multicolumn{6}{|l|}{ Race/ethnicity (\%) } \\
\hline African Amer. & 70 & 36.5 & 34.9 & \multirow[t]{4}{*}{$5.8(4)$} & 39.0 \\
\hline Caucasian & 30 & 57.7 & 53.5 & & 53.3 \\
\hline Other & 0 & 5.8 & 11.6 & & 7.6 \\
\hline$n$ & 10 & 52 & 43 & & $105^{\mathrm{a}}$ \\
\hline \multicolumn{6}{|l|}{ Employment status (\%) } \\
\hline Not working & 50 & 42.6 & 51.2 & \multirow[t]{3}{*}{$0.70(2)$} & 46.9 \\
\hline Working (part or full time) & 50 & 57.4 & 48.8 & & 53.1 \\
\hline$n$ & 10 & 47 & 41 & & $98^{\mathrm{b}}$ \\
\hline \multicolumn{6}{|l|}{ Education } \\
\hline Any college & 80 & 71.7 & 78 & \multirow[t]{3}{*}{$0.60(2)$} & 75.3 \\
\hline No college & 20 & 28.3 & 22 & & 24.7 \\
\hline$n$ & 10 & 46 & 41 & & $97^{\mathrm{c}}$ \\
\hline \multicolumn{6}{|l|}{ Income } \\
\hline$\$ 15,000$ and below & 70 & 41.3 & 40 & \multirow[t]{3}{*}{$3.14(2)$} & 43.8 \\
\hline Above $\$ 15,000$ & 30 & 58.7 & 60 & & 56.3 \\
\hline$n$ & 10 & 46 & 40 & & $96^{\mathrm{d}}$ \\
\hline Age- $M(S D)$ & $33.7(8.3)$ & $32.1(10.1)$ & $30.9(9.5)$ & $F(2,100)=.52$ & \\
\hline$n$ & 16 & 46 & 39 & & $101^{\mathrm{e}}$ \\
\hline
\end{tabular}

Some marginal means do not sum to 100 due to rounding.

a 2 not reported.

b9 not reported.

${ }^{\mathrm{c}} 10$ not reported.

${ }^{\mathrm{d}} 11$ not reported.

${ }^{\mathrm{e}} 6$ not reported. 
symptoms and treatment for the past month. The original measure assessed only the frequency of 54 items and did not include a specific time constraint for the symptoms. Items were summed to obtain a total score. Internal consistency is high for both the original scale with Cronbach's $\alpha$ of .91 $1^{[22]}$ and our modification with a Cronbach's $\alpha$ of .94. Test-retest reliability is also good with a correlation of .83 over a 2 month period. ${ }^{[22]}$ The current version added items to assess physical symptoms not assessed by the original PILL that are relevant to our population (such as urinary or female sexual organ difficulties and problems related to physical injuries). To make the measure more amenable to repeated assessments, the time of 1 month was allotted for the symptoms. In our modified version, participants were also asked how frequently they have experienced the item in the past month $(0=$ not at all, $5=$ more than once per day). For those items endorsed, they were then asked how much discomfort they experienced $(0=$ no discomfort, $5=$ severe discomfort $)$, whether they visited a medical professional, and whether they received treatment. Due to inconsistent reporting of doctor's visits and treatment, our analyses focus on just the frequency and discomfort scales. For each patient at each time point, an average frequency and discomfort were calculated. The average frequency was calculated over all 77 items. The average discomfort was scored as the average of all items that had been endorsed.

PTSD Symptom Scale-Interview (PSS-I). The PSS-I ${ }^{[23]}$ is a semistructured interview that consists of 17 items corresponding to the DSM-IV PTSD symptoms. Items are rated on 0-3 scales for combined frequency and severity in the past 2 weeks. Inter-rater reliability for PTSD diagnosis $(\kappa=.91)$ and overall severity $(r=.97)$ are excellent. ${ }^{[23]}$ As reported in the main outcome study, $5 \%$ of the audiotaped PSS-I interviews in this study were randomly selected for rating by a second evaluator. The inter-rater reliability was $.94 .^{[18]}$

Beck Depression Inventory (BDI). The $\mathrm{BDI}^{[24]}$ is a 21 -item self-report inventory measuring depression severity. Split-half reliability was .93 . Correlations with clinician ratings ranged from .62-.66.

Social Adjustment Scale (SAS). The SAS ${ }^{[20]}$ is a semistructured interview assessing functioning in eight specific areas on separate 7point scales, with higher scores indicating more severe maladjustment. We used only the Global scale.

\section{ANALYSIS}

To examine change from pretreatment to posttreatment for each of the three outcome measures (PILL frequency, PILL discomfort, and Global SAS), three separate mixed analyses of variance (ANOVAs) were conducted with treatment group (WL, PE, PE/CR) as the independent variable and the pretreatment and posttreatment scores as the repeated dependent measures. Significant main effects/interactions were then followed up with appropriate tests. To examine change in the follow-up period among all active treatment completers for each of the three outcome measures (PILL frequency, PILL discomfort, and Global SAS), three mixed ANOVAs were conducted using LOCF with treatment group (PE, PE/CR) as the independent variable and 3-, 6-, and 12-month scores as the repeated dependent measures. Significant main effects/interactions were then followed up with appropriate tests.

Due to the shortcomings of raw change scores (i.e., capitalization on monomethod variance, overestimation and underestimation of change), standardized residual gain scores were calculated for each of these measures to examine the relationships between pretreatment and posttreatment change in frequency and discomfort of reported health difficulties, global social functioning, and PTSD and depression severity. Pearson correlations of these change scores were then calculated (Table 3). To more closely examine the contributions of change in PTSD and depression to change in each of the outcome measures with significant change (frequency of reported health problems and global social functioning), two separate regression analyses were conducted with change in the outcome measure simultaneously regressed on change in PTSD and change in depression.

\section{RESULTS}

Means and standard deviations for the health measures and general social functioning at each time point are presented in Table 2. When examining change in frequency of reported health problems from pretreatment to posttreatment, the main effect for time $[F \quad(1,104)=64.8, \quad P<.0001]$ was significant but qualified by an interaction with treatment group $[F$ $(2,104)=3.96, P=.02]$. The main effect of treatment group was not significant $[F(2,104)=1.03$, ns $]$. An ANOVA within each time point was conducted to clarify the significant interaction. At pretreatment, groups did not differ in frequency of reported health problems, $F(2,104)=.04$, ns. At posttreatment, the groups significantly differed in frequency of reported health problems, $F(2,104)=3.96, P=.02$. Follow-up pair wise comparisons using least significant difference corrections demonstrate that the wait list group $(M=0.72, S D=0.54)$ reported significantly more frequent physical symptoms than either the $\mathrm{PE}$ $(M=0.44, \quad S D=0.31, \quad$ mean difference $=0.28$, $P=0.01)$ or $\mathrm{PE} / \mathrm{CR}(M=0.43, S D=0.43$, mean difference $=0.30, P=.01)$ groups. The two active treatment groups did not differ.

When examining changes in frequency of reported health problems during follow-up, both the main effect for time $[F(2,172)=2.18, \mathrm{~ns}]$ and treatment group $[F$ $(1,86)=.01$, ns $]$ as well as the interaction $[F(2$, $172)=.24$, ns] were not significant.

Similarly, when examining change in physical health discomfort from pretreatment to posttreatment, the main effect for time $[F(1,99)=.07, \mathrm{~ns}]$ and treatment group $[F(2,99)=2.37, P=.10]$ as well as the interaction $[F(2,99)=1.33$, ns] were not significant.

When examining changes in physical health discomfort during follow-up, both the main effect for time $[F(2,164)=2.78, \mathrm{~ns}]$ and treatment group $[F(1$, $82)=.39, \mathrm{~ns}]$ as well as the interaction $[F(2$, $164)=1.36$, ns] were not significant.

When examining change in global social functioning from pretreatment to posttreatment, both the main effect for time $[F(1,101)=30.4, P<.0001]$ and 
TABLE 2. Means and standard deviations of health perceptions and global social function across time

\begin{tabular}{|c|c|c|c|c|}
\hline & & $W L$ & $\mathrm{PE}$ & $\mathrm{PE} / \mathrm{CR}$ \\
\hline \multirow[t]{10}{*}{ PILL Frequency } & Pre mean (SD) & $0.86(.52)$ & $0.85(.53)$ & $0.82(.53)$ \\
\hline & $n=107$ & $n=19$ & $n=48$ & $n=40$ \\
\hline & Post mean (SD) & $0.72(.54)$ & $0.44(.31)$ & $0.42(.43)$ \\
\hline & $n=107$ & $n=19$ & $n=48$ & $n=40$ \\
\hline & 3 Mo LOCF mean (SD) & - & $0.42(.33)$ & $0.41(.40)$ \\
\hline & $n=88$ & & $n=48$ & $n=40$ \\
\hline & 6 Mo LOCF mean (SD) & - & $0.36(.36)$ & $0.37(.37)$ \\
\hline & $n=88$ & & $n=48$ & $n=40$ \\
\hline & 12 Mo LOCF mean (SD) & - & $0.39(.36)$ & $0.37(.38)$ \\
\hline & $n=88$ & & $n=48$ & $n=40$ \\
\hline \multirow[t]{10}{*}{ PILL Discomfort } & Pre mean (SD) & $2.33(.89)$ & $2.08(.83)$ & $2.07(.72)$ \\
\hline & $n=102$ & $n=18$ & $n=45$ & $n=39$ \\
\hline & Post mean (SD) & $2.55(1.02)$ & $2.07(.84)$ & $1.92(.77)$ \\
\hline & $n=102$ & $n=18$ & $n=45$ & $n=39$ \\
\hline & 3 Mo LOCF mean (SD) & - & $216(1.04)$ & $2.15(.92)$ \\
\hline & $n=84$ & & $n=45$ & $n=39$ \\
\hline & 6 Mo LOCF mean (SD) & - & $1.99(.84)$ & $1.93(.88)$ \\
\hline & $n=84$ & & $n=45$ & $n=39$ \\
\hline & 12 Mo LOCF Mean (SD) & - & $2.17(.95)$ & $1.90(.87)$ \\
\hline & $n=84$ & & $n=45$ & $n=39$ \\
\hline \multirow[t]{10}{*}{ Global SAS } & Pre MEAN (SD) & $4.11(0.76)$ & $3.90(0.72)$ & $3.75(0.78)$ \\
\hline & $n=106$ & $n=18$ & $n=46$ & $n=40$ \\
\hline & Post mean (SD) & $3.89(1.37)$ & $2.96(1.07)$ & $2.90(1.06)$ \\
\hline & $n=104$ & $n=18$ & $n=46$ & $n=40$ \\
\hline & 3 Mo LOCF Mean (SD) & - & $2.71(1.20)$ & $2.85(1.08)$ \\
\hline & $n=88$ & & $n=48$ & $n=40$ \\
\hline & 6 Mo LOCF Mean (SD) & - & $2.65(1.14)$ & $2.68(1.19)$ \\
\hline & $n=88$ & & $n=48$ & $n=40$ \\
\hline & 12 Mo LOCF Mean(SD) & - & $2.52(1.03)$ & $2.50(1.13)$ \\
\hline & $n=88$ & & $n=48$ & $n=40$ \\
\hline
\end{tabular}

PILL, Pennebaker Inventory of Limbic Languidness; SAS, Social Adjustment Sclale; LOCF, last observation carried forward.

treatment group $[F(2,101)=5.09, P=.008]$ were significant. The interaction approached significance $[F$ $(2,101)=2.61, P=.08]$. An ANOVA within each time point was conducted to clarify the main effects. At pretreatment, groups did not differ in global social functioning, $F(2,105)=1.47$, ns. At posttreatment, the groups significantly differed in global social functioning, $F(2,104)=5.18, P=.007$. Follow-up pair wise comparisons using least significant difference corrections demonstrate that the wait list group $(M=3.84$, $S D=1.34)$ reported significantly worse global social functioning than either the $\mathrm{PE}(M=2.96, S D=1.07$, mean difference $=0.89, P=.005)$ or PE/CR $(M=2.90$, $S D=1.06$, mean difference $=0.94, P=0.003)$ groups at posttreatment. The two active treatment groups did not differ.

When examining changes in global social functioning during follow-up, the main effect for time $[F(2$, $172)=4.17, P=.02]$ was significant. Both the main effect of treatment group $[F(1,86)=.05, \mathrm{~ns}]$ and the interaction $[F(4,208)=.23$, ns $]$ were not significant. Follow-up pair wise comparisons demonstrated significant continued improvement in global social function such that that at 12-month follow-up $(M=2.51, S D=1.07)$ social functioning was better than at 3 -month follow-up, $(M=2.77, S D=1.14$; $t(87)=2.68, P=.009)$. The other time points do not significantly differ.

Table 3 presents the Pearson correlations between change in PTSD, depression, social functioning, and frequency and discomfort of reported health difficulties. All of these correlations except between global social functioning and physical health discomfort were significant. The magnitude of correlations between PTSD and depressive symptoms and both frequency of reported physical health difficulties and global social functioning were consistently higher than correlations between symptoms and physical health discomfort. However, this difference was not statistically significant.

In a regression to examine the contributions of change in PTSD and depression on change in frequency of reported physical health difficulties, the overall model was significant, $F(2,105)=22.1$, $P<.0001$, Adjusted $R^{2}=.29, \mathrm{SEE}=.87$. However, only change in PTSD significantly contributed to the model (PTSD, $B=.39, S E=.12, P=.001$; Depression, $B=.23, S E=.12, P=.06)$. Collinearity diagnostics suggest that change in PTSD and depression is significantly collinear (Model Conditional 
TABLE 3. Pearson correlations between pre to post treatment change in PTSD severity, depression severity, frequency and discomfort of physical health problems, and global social functioning

\begin{tabular}{|c|c|c|c|c|}
\hline & PILL frequency $r(n)$ & PILL discomfort $r(n)$ & SAS $r(n)$ & PSSI $r(n)$ \\
\hline PILL frequency & - & - & - & - \\
\hline PILL discomfort & $.37(102)^{* *}$ & - & - & - \\
\hline SAS & $.26(86)^{*}$ & $.12(82)$ & - & - \\
\hline PSSI & $.53(106)^{* *}$ & $.23(101)^{*}$ & $.50(86)^{* *}$ & - \\
\hline BDI & $.47(107)^{* *}$ & $.22(102)^{*}$ & $.47(86)^{* *}$ & $.70(106)^{* *}$ \\
\hline
\end{tabular}

${ }^{*} p<.05,{ }^{* *} p<.01$.

PILL, Pennebaker Inventory of Limbic Languidness; SAS, Social Adjustment Sclale.

Index $=2.4$; Variance proportions: Depression $=.85$ and PTSD $=.85$ ).

In a regression to examine the contributions of change in PTSD and depression on change in global social functioning, the overall model was significant, $F$ $(2,85)=19.2, P<.0001$, Adjusted $R^{2}=.30, \mathrm{SEE}=.83$. Both change in PTSD and depression significantly contributed to the model (PTSD, $B=.47, S E=.14$, $P=.001 ;$ Depression, $B=.35, S E=.13, P=.007)$. Collinearity diagnostics suggest that change in PTSD and depression are significantly collinear (Model Conditional Index $=2.1$; Variance proportions: Depression $=.71$ and PTSD $=.80$ ).

\section{DISCUSSION}

Support for our primary hypothesis regarding reductions in self-reported physical health perceptions was mixed. In support, the frequency of reported physical health difficulties was significantly reduced in the $\mathrm{PE}$ and $\mathrm{PE} / \mathrm{CR}$ conditions compared to the waitlist condition and these reductions did not demonstrate significant change during the 12-month follow-up period. However, discomfort associated with reported physical health difficulties did not demonstrate significant change over treatment and no difference was detected between the active treatment and waitlist conditions. As such, it appears that though treatment may have resulted in improvement in physical health through reduction in the reported frequency of problems, the problems that continued to be reported at posttreatment remained moderately severe in discomfort (just over 2 on a $0-5$ scale).

Our hypothesis concerning improvements in general social functioning over active treatment was supported. Both the $\mathrm{PE}$ and $\mathrm{PE} / \mathrm{CR}$ groups reported improved social functioning at posttreatment compared to the waitlist. Contrary to our hypothesis, additional improvement in general social functioning was found between 3- and 12-month follow-up assessments. This pattern suggests that changes in functioning among women receiving PE for PTSD may continue even after therapy has concluded. This is contrary to other studies that have tended to generally support a pattern of maintenance of gains in areas of symptom reduction. ${ }^{[18,25]}$ It is possible that this general social functioning measure may be picking up on larger changes that may take time to become apparent following therapy. Together, these findings suggest that improvements in exposure-based treatment of PTSD go well beyond traditional mental health factors.

Correlational and additional regression analyses strongly support that changes in PTSD and depression are related to changes in reported physical health difficulties and general social functioning. More specifically, changes in PTSD and depressive symptoms account for $29 \%$ of the variance in change in frequency of reported health problems over treatment and $30 \%$ of the variance in change in general social functioning. Importantly, only changes in PTSD symptoms significantly contribute to the model predicting change in physical health problems with depression associated only at a trend level. However, collinearity between these measures makes it difficult to assess the separate contributions of PTSD and depression and suggests that both factors are likely important. Despite high collinearity in the model predicting change in social functioning, changes in both depression and PTSD significantly contribute. Although we cannot determine whether the physical or mental health factor chronologically occurs first due to simultaneous assessment, this does suggests that both factors are important to consider when assessing improvement in PTSD treatment.

Several caveats are apparent. First, this study is a secondary analysis of data collected for a larger outcome study. As such, data are not available for patients who dropped out. Although LOCF analyses were conducted with study completers to conservatively examine the data, it is possible that a different pattern may be apparent if data were available from women who dropped out. Second, we used a modification of the standard PILL in that items were added that are specifically relevant to a female assault population and the scoring was modified to include a 1-month time limit. Although we reported reliability of the frequency data in our sample, norms for this modification of the measure are not available and previous norms would not apply due to the change in number of items. Third, our primary measure is self-report. We do not have medical data to determine whether there 
was an actual change in medical service use or medical problem severity. However, these data support that closer examination of change in physical health and health perceptions is warranted. Lastly, these analyses were conducted on an all female sample of sexual assault survivors. Whether these results generalize to male samples or other trauma populations is unclear.

\section{CONCLUSION}

$\mathrm{PE}$ resulted in significant reductions from pretreatment to posttreatment in frequency of reported health difficulties that were maintained over 1-year of followup. These reductions were greater than changes in a waitlist group. In addition, PE resulted in improvement in social functioning from pretreatment to posttreatment and continued gains were found during the 1-year follow-up. Together these findings support the broad impact of PE on patient functioning beyond PTSD and other mental health symptoms.

Acknowledgments. This study was supported by a National Institute of Mental Health grant (MH42178) awarded to Edna B. Foa.

\section{REFERENCES}

1. Breslau N, Davis GC, Andreski P, Peterson E. Traumatic events and posttraumatic stress disorder in an urban population of young adults. Arch Gen Psychiatry 1991;48:216-222.

2. Kessler RC, Sonnega A, Bromet E, Hughes M. Posttraumatic stress disorder in the National Comorbidity Survey. Arch Gen Psychiatry 1995;52:1048-1060.

3. Norris FH. Epidemiology of trauma: Frequency and impact of different potentially traumatic events on different demographic groups. J Consul Clin Psychol 1992;60:409-418.

4. Resnick HS, Kilpatrick DG, Dansky BS, Saunders BE, Best CL. Prevalence of civilian trauma and posttraumatic stress disorder in a representative national sample of women. J Consult Clin Psychol 1993;61:984-991.

5. Solomon SD, Davidson JRT. Trauma: prevalence, impairment, service use, and cost. Journal of Clinical Psychiatry 1997;58:5-11.

6. Green BL, Kimerling R. Trauma, posttraumatic stress disorder, and health status. In: Schnurr PPG, Bonnie L., eds. Trauma and Health: Physical Health Consequences of Exposure to Extreme Stress. Washington, DC: American Psychological Association; 2004; 13-42.

7. Kimerling R, Clum GA, Wolfe J. Relationships among trauma exposure, chronic posttraumatic stress disorder symptoms, and self-reported health in women: Replication and extension. J Traum Stress 2000;13:115-128.

8. Lauterbach D, Vora R, Rakow M. The relationship between posttraumatic stress disorder and self-reported health problems. Psychosom Med 2005;67:939-947.
9. Kimerling R. An investigation of sex differences in nonpsychiatric morbidity associated with posttraumatic stress disorder. J Am Med Womens Assoc 2004;59:43-47.

10. Zoellner LA, Goodwin ML, Foa EB. PTSD severity and health perceptions in female victims of sexual assault. J Trauma Stress 2000;13:635-649.

11. Rauch SAM, Morales KH, Zubritsky C, Knott K, Oslin D. Posttraumatic stress, depression, and health among older adults in primary care. Am J Geriatr Psychiatry 2006;14:316-324.

12. Richardson JD, Elhai JD, Pedlar DJ. Association of PTSD and Depression With Medical and Specialist Care Utilization in Modern Peacekeeping Veterans in Canada With Health-Related Disabilities. J Clin Psychiatry 2006;67: 1240-1245.

13. Schnurr PP, Green BL. Understanding Relationships Among Trauma, Post-Tramatic Stress Disorder, and Health Outcomes. Adv Mind Body Med 2004;20:18-29.

14. Clum GA, Calhoun KS, Kimerling R. Associations among symptoms of depression and posttraumatic stress disorder and self-reported health in sexually assaulted women. J Nerv Men Dis 2000;188:671-678.

15. Wagner AW, Wolfe J, Rotnitsky A, Proctor SP, Erickson DJ. An investigation of the impact of posttraumatic stress disorder on physical health. J Trauma Stress 2000;13:41-55.

16. Baron RM, Kenny DA. The moderator-mediator variable distinction in social psychological research: conceptual, strategic, and statistical considerations. J Pers and Soc Psychol 1986; 51:1173-1182.

17. Schnurr PP, Spiro A, III. Combat disorder, posttraumatic stress disorder symptoms, and health behaviors as predictors of selfreported physical health in older veterans. J Nerv Men Dis 1999;187:353-359.

18. Foa EB, Hembree EA, Cahill SP et al. Randomized trial of prolonged exposure for posttraumatic stress disorder with and without cognitive restructuring: outcome at academic and community clinics. J Consult Clin Psychol 2005;73:953-964.

19. Hembree EA, Foa EB, Dorfan NM, Street GP, Kowalski J, Tu X. Do patients drop out prematurely from exposure therapy for PTSD? J Trauma Stress 2003;16:555-562.

20. Weissman MM, Paykel ES. The Depressed Woman: A Study of Social Relationships. Oxford, England: U Chicago Press; 1974.

21. IOM. 2007. Treatment of PTSD: An assessment of the the evidence. Washington, DC: Institute of Medicine.

22. Pennebaker JW. 1982. The Pennebaker Inventory of Limbic Languidness (the PILL). In: Pennebaker JW, ed. The Psychology of Physical Symptoms. New York, NY: Springer-Verlag.

23. Foa EB, Riggs DS, Dancu CV, Rothbaum BO. Reliability and validity of a brief instrument for assessing post-traumatic stress disorder. J Trauma Stress 1993;6:459-473.

24. Beck AT, Ward CH, Mendelson M, Mock J, Erbaugh J. An inventory for measuring depression. Arch Gen Psychiatry 1961; 4:561-571.

25. Foa EB, Dancu CV, Hembree EA, Jaycox LH, Meadows EA, Street GP. A comparison of exposure therapy, stress inoculation training, and their combination for reducing posttraumatic stress disorder in female assault victims. J Consult Clin Psychol 1999; 67:194-200. 